\title{
Engineering limonene and bisabolene production in wild type and a glycogen-deficient mutant of Synechococcus sp. PCC 7002
}

\author{
Fiona K. Davies ${ }^{1}$, Victoria H. Work ${ }^{2}$, Alexander S. Beliaev ${ }^{3}$ and Matthew C. Posewitz ${ }^{1}$ \\ 1 Department of Chemistry and Geochemistry, Colorado School of Mines, Golden, CO, USA \\ ${ }^{2}$ Civil and Environmental Engineering Division, Colorado School of Mines, Golden, CO, USA \\ ${ }^{3}$ Biological Sciences Division, Pacific Northwest National Laboratory, Richland, WA, USA
}

\section{Edited by:}

Anne M. Ruffing, Sandia National Laboratories, USA

Reviewed by:

Weiwen Zhang, Tianjin University, China

Taek Soon Lee, Lawrence Berkeley National Laboratory, USA

*Correspondence:

Fiona K. Davies, Department of

Chemistry and Geochemistry,

Colorado School of Mines, 1500

Illinois Street, Golden, CO 80401,

USA

e-mail:fdavies@mines.edu

\begin{abstract}
The plant terpenoids limonene $\left(\mathrm{C}_{10} \mathrm{H}_{16}\right)$ and $\alpha$-bisabolene $\left(\mathrm{C}_{15} \mathrm{H}_{24}\right)$ are hydrocarbon precursors to a range of industrially relevant chemicals. High-titer microbial synthesis of limonene and $\alpha$-bisabolene could pave the way for advances in in vivo engineering of tailor-made hydrocarbons, and production at commercial scale. We have engineered the fast-growing unicellular euryhaline cyanobacterium Synechococcus sp. PCC 7002 to produce yields of $4 \mathrm{mg} \mathrm{L}^{-1}$ limonene and $0.6 \mathrm{mg} \mathrm{L}^{-1} \alpha$-bisabolene through heterologous expression of the Mentha spicata L-limonene synthase or the Abies grandis (E)- $\alpha$-bisabolene synthase genes, respectively. Titers were significantly higher when a dodecane overlay was applied during culturing, suggesting either that dodecane traps large quantities of volatile limonene or $\alpha$-bisabolene that would otherwise be lost to evaporation, and/or that continuous product removal in dodecane alleviates product feedback inhibition to promote higher rates of synthesis. We also investigate limonene and bisabolene production in the $\Delta g l g C$ genetic background, where carbon partitioning is redirected at the expense of glycogen biosynthesis. The Synechococcus sp. PCC $7002 \Delta g / g C$ mutant excreted a suite of overflow metabolites ( $\alpha$-ketoisocaproate, pyruvate, $\alpha$-ketoglutarate, succinate, and acetate) during nitrogen-deprivation, and also at the onset of stationary growth in nutrient-replete media. None of the excreted metabolites, however, appeared to be effectively utilized for terpenoid metabolism. Interestingly, we observed a 1.6- to 2.5-fold increase in the extracellular concentration of most excreted organic acids when the $\Delta g / g C$ mutant was conferred with the ability to produce limonene. Overall, Synechococcus sp. PCC 7002 provides a highly promising platform for terpenoid biosynthetic and metabolic engineering efforts.
\end{abstract}

Keywords: terpenoids, limonene, bisabolene, cyanobacteria, glycogen, $g l g C$, metabolic sink

\section{INTRODUCTION}

In recent years, cyanobacteria have been successfully used as a platform to generate a range of commercially significant products, including isoprene (Lindberg et al., 2010; Bentley and Melis, 2012; Bentley et al., 2014), isobutanol (Atsumi et al., 2009), 2,3butanediol (Oliver et al., 2013, 2014), and ethylene (Ungerer et al., 2012). In all cases, photoassimilated carbon was drawn from native metabolic pathways through the heterologous expression of one or more enzymes to create new carbon sinks. While demonstrating the proof-of-principle, these strategies are unlikely to attain high yields of novel end-products as carbon partitioning and regulation are hardwired into the organism's central metabolic networks. For cyanobacteria, one of the main metabolic engineering bottlenecks is the ability to redirect carbon from the major glycogen and protein sinks to pathways of interest. A careful rewiring of central metabolism is required to increase product yield at the expense of biomass accumulation, without negatively influencing photosynthesis and carbon fixation rates.

In the context of photoautotrophic biotechnology, the terpenoid biosynthetic pathway is of particular interest, as it produces the largest and most diverse array of naturally occurring organic compounds (typically of plant origin) (Davies et al., 2014). The plant terpenoids limonene $\left(\mathrm{C}_{10} \mathrm{H}_{16}\right)$ and bisabolene $\left(\mathrm{C}_{15} \mathrm{H}_{24}\right)$ are recognized as precursors to a range of commercially valuable products, with applications in biofuels, bioplastics, pharmaceutical, nutraceutical, and cosmetic industries (Duetz et al., 2003; Peralta-Yahya et al., 2011). In this study, we engineer the fastgrowing euryhaline cyanobacterium Synechococcus sp. PCC 7002 for the production of limonene and $\alpha$-bisabolene through heterologous expression of the Mentha spicata L-limonene synthase and Abies grandis (E)- $\alpha$-bisabolene synthase genes. In cyanobacteria, terpenoids are synthesized via the 2-C-methyl-D-erythritol 4-phosphate (MEP) pathway, utilizing photosynthetically generated pyruvate and glyceraldehyde-3-phosphate (GAP) as the primary substrates (Figure 1). Limonene and bisabolene represent new carbon sinks that draw from the native terpenoid pathway at the levels of geranyl pyrophosphate (GPP) and farnesyl pyrophosphate (FPP).

The two major competing pathways for cyanobacterial terpenoid production are protein and glycogen (the cyanobacterial 


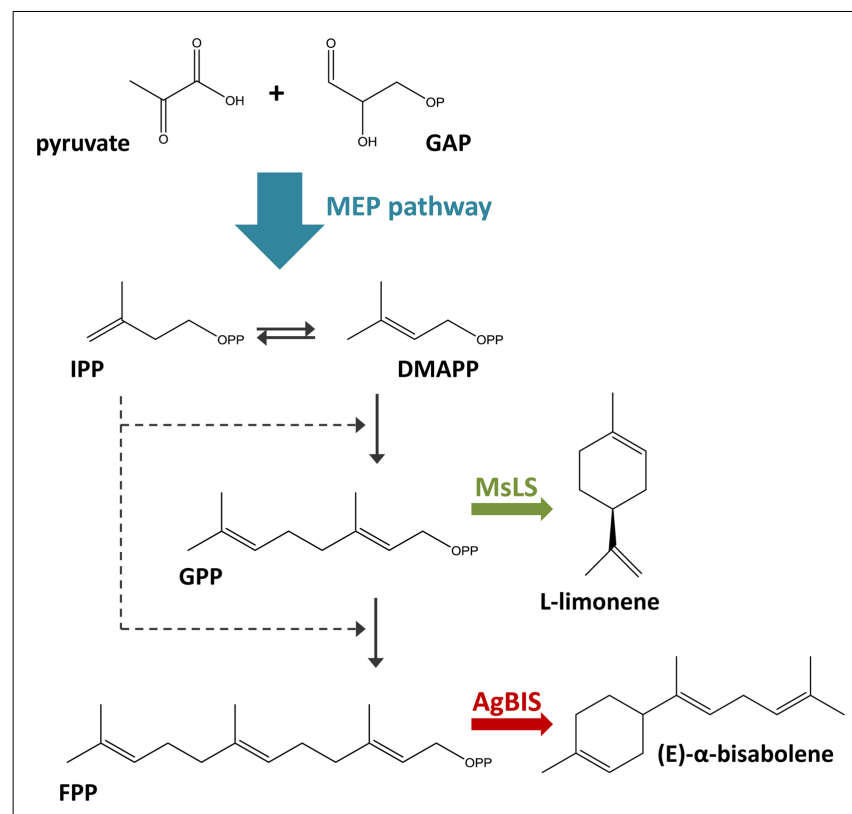

FIGURE 1 |Terpenoid biosynthesis via the 2-C-methyl- - -erythritol 4-phosphate (MEP) pathway. The MEP terpenoid biosynthetic pathway converts pyruvate and glyceraldehyde-3-phosphate (GAP) feedstock to the C5 isomers, isopentenyl pyrophosphate (IPP), and dimethylallyl pyrophosphate (DMAPP). The stepwise addition of IPP to DMAPP generates C10 geranyl pyrophosphate (GPP) and C15 farnesyl pyrophosphate (FPP), the respective precursors to monoterpenes and sesquiterpenes. In this study, the heterologous expression of the $M$. spicata L-limonene synthase (MsLS) and $A$. grandis (E)- $\alpha$-bisabolene synthase (AgBIS) in Synechococcus sp. PCC 7002 was designed to draw on GPP and FPP pools, respectively, generated by the native cyanobacterial MEP pathway for the production of the non-native terpenoids L-limonene and $\alpha$-bisabolene.

storage carbohydrate) biosynthesis. Recent studies in Synechocystis sp. PCC 6803 and Synechococcus sp. PCC 7942 have demonstrated that upon simultaneous inactivation of protein and glycogen sinks ( $v i a$ nitrogen-deprivation in the $\Delta g l g C$ glycogen-deficient mutant), a suite of organic acids are excreted due to the resulting metabolic imbalance (Carrieri et al., 2012; Grundel et al., 2012; Hickman et al., 2013). In our study, we sought to elucidate how such major shifts in flux distribution will affect the overall metabolic partitioning toward the terpenoid pathway in Synechococcus sp. PCC 7002, using the newly engineered limonene and $\alpha$-bisabolene terpenoid sinks as the reporting products.

\section{MATERIALS AND METHODS CLONING AND PLASMID CONSTRUCTION}

The E. coli $\mathrm{DH} 5 \alpha$ strain was used for routine subcloning according to standard protocols. The (-)-4S-limonene synthase gene from M. spicata (GenBank accession Q40322) (Colby et al., 1993) was optimized for codon-usage in Synechococcus sp. PCC 7002 (DNA 2.0, USA) without the predicated chloroplast transit peptide (cTP), and is referred to in this work as MsLS. The first 168 bases of Q40322 encoding the cTP were replaced with an ATG codon for translation initiation in Synechococcus sp. PCC 7002, retaining residues R58/R59 that are required for catalytic activity (Williams et al., 1998). The (E)- $\alpha$-bisabolene synthase gene from A. grandis (GenBank accession O81086) (Bohlmann et al., 1998; Trapp and Croteau, 2001) was also optimized for codon-usage in Synechococcus sp. PCC 7002 (DNA 2.0, USA), and is referred to as AgBIS. Oligonucleotide primers used to amplify these codon-optimized genes are as follows (introduced restriction sites for cloning are indicated in bold, and lower case letters represent start or stop codons): MsLS, MsLS_NcoI_F, 5' CTTCGCTGAACCatgGAACGTCGCTC-3' ${ }^{\prime}$, and MsLS_BamHI_R, 5'-TGACGGGATCCttaAGCGAAGG-3'; AgBIS, AgBIS_NcoI_F, 5'-AGATTAATTCCatgGCCGGTGTGA-3' ; and AgBIS_BamHI_R, $5^{\prime}$-GCCGGATCCttaTAACGGCAAC-3'.

A neutral site (NSI) for the integration of transgenes was identified in the Synechococcus sp. PCC 7002 genome between two open-reading frames encoding hypothetical proteins (SYNPCC7002_A0935 and SYNPCC7002_A0936), which we have termed NSI. This was used as the site of integration for the limonene synthase (MsLS) and $\alpha$-bisabolene synthase (AgBIS) genes. Oligonucleotide primers designed to amplify $750 \mathrm{bp}$ up-stream (us) and down-stream (ds) regions flanking NSI, which were used for double homologous recombination in Synechococcus sp. PCC 7002 are as follows (introduced restriction sites are indicated in bold): NSIus, 935us_NsiI_F, 5'-AGTTCACATGCATAAAGTCAA-3' , and 935us_EcoRI_R, 5' CGTATAGGAATTCTTACTCAG-3'; NSIds, 936ds_SalI_F, 5'GCATACTGTCGACCTATTTTAT-3'; and 936ds_SphI_R, 5' AGTTGACGCATGCAGAGGTGG-3'. These regions were cloned into a pAQ1 plasmid containing the Synechocystis sp. PCC 6803 $c p c B A$ (cpc) promoter ( $\mathrm{Xu}$ et al., 2011). The MsLS and AgBIS transgenes, along with a spectinomycin-resistance cassette (SmR), were cloned between the NSI-flanking regions immediately downstream of the $c p c$ promoter, generating plasmids pNSI-cpc-MsLSSmR and pNSI-cpc-AgBIS-SmR. To demonstrate that any phenotypes arising from the expression of MsLS or AgBIS were not a direct result of interference with the NSI region of Synechococcus sp. PCC 7002 genomic DNA, we also constructed the control plasmid pNSI-cpc-YFP-SmR, containing a YFP gene in place of the MsLIM or AgBIS terpene synthase (TPS) genes.

\section{CYANOBACTERIAL STRAINS, GROWTH CONDITIONS, AND TRANSFORMATION}

Wild type and transformant Synechococcus sp. PCC 7002 strains were maintained on solid $\mathrm{A}^{+}$media (Stevens et al., 1973) supplemented with $8.25 \mathrm{mM}$ Tris- $\mathrm{HCl}(\mathrm{pH} 8.2)$ and $0.3 \%$ sodium thiosulfate. Where appropriate, spectinomycin was used at a concentration of $50 \mu \mathrm{g} \mathrm{mL}^{-1}$ and kanamycin at $100 \mu \mathrm{g} \mathrm{mL}^{-1}$. Liquid cultures were grown in $\mathrm{A}^{+}$media supplemented with $8.25 \mathrm{mM}$ Tris$\mathrm{HCl}$ ( $\mathrm{pH}$ 8.2). For nitrogen-deprivation, $\mathrm{NaNO}_{3}$ was omitted from the growth media and replaced mol:mol with $\mathrm{NaCl}$. Nitrogendeplete media is referred to as $\mathrm{A}^{+}(-\mathrm{N})$. Cultures were grown in $250 \mathrm{~mL}$ Erlenmeyer flasks on an orbital shaker at $37^{\circ} \mathrm{C}$, in an incubator with an atmosphere of $1 \% \mathrm{CO}_{2}$ in air, and constant illumination at $250 \mu \mathrm{mol}$ photons $\mathrm{m}^{-2} \mathrm{~s}^{-1}$ of photosynthetically active radiation (PAR). Liquid starter cultures were grown in the presence of antibiotics; however, during experimental procedures antibiotics were eliminated. Transformation of Synechococcus sp. PCC 7002 with the plasmid constructs described above was performed 
according to established procedures for cyanobacteria (Eaton-Rye, 2011) to generate the strains LS (containing MsLS) and BIS (containing AgBIS). Complete chromosomal segregation for the introduced transgenes was achieved through propagation of multiple generations on selective agar and verified by colony-PCR using the primers: NSI_us_F, 5'-CTAGCACAAATGAAGCCCGAC-3' , and NSI_ds_R, 5'-GCAGATATAAGCAACGGTACAG-3' (Figure 2). The $\Delta g \lg C$ strain was obtained from D. A. Bryant, which was generated via the insertional disruption of the $g \lg C$ open reading frame (SYNPCC7002_A0095) with a kanamycin-resistance cassette (KmR) (Guerra et al., 2013). Oligonucleotide primers used to verify $\operatorname{glgC}$ disruption in transformant strains are: glgC_F, 5' -TCACGTAGTCGGGTTTGATGTC-3' , and glgC_R, 5' CACTAAAGTCCACGACACGACC- $3^{\prime}$. The $\Delta g \operatorname{lgC}$ strain was also transformed with the MsLS and AgBIS plasmid constructs to generate strains $\Delta g l g C: \mathrm{LS}$ and $\Delta g \operatorname{lgC}$ :BIS, respectively.

\section{TERPENOID EXTRACTION AND QUANTIFICATION}

Synechococcus cultures used for terpenoid production assays were grown photoautotrophically in $100 \mathrm{~mL}$ batches, with an inoculation density of $\mathrm{OD}_{730 \mathrm{~nm}}=2.0$. Where indicated, a $5-\mathrm{mL}$ dodecane (analytical grade, Sigma-Aldrich, USA) overlay was applied to the culture at $t=0$ to trap terpenoids during the 96-h experimental time course. At $96 \mathrm{~h}$, cells were harvested by centrifugation to separate the biomass from the media. Terpenoid extraction was performed on the cell pellet using the chloroform:methanol:water

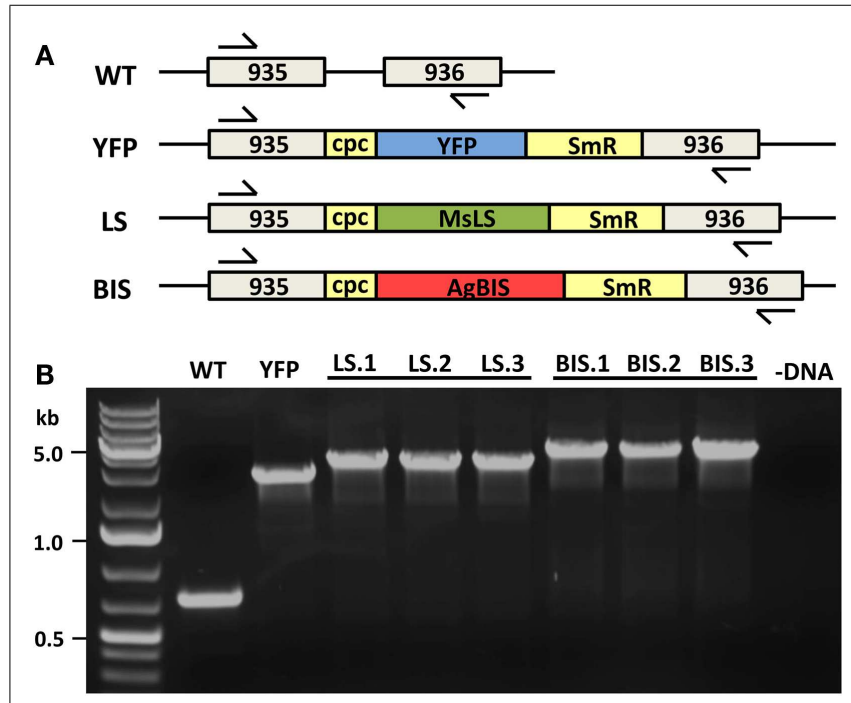

FIGURE 2 | MsLS and AgBIS transgene integration to the Synechococcus chromosome. (A) The YFP, MsLS, and AgBIS transgene constructs were introduced to a neutral site (NSI) of genomic DNA (between open-reading frames A0935 and A0936) via double homologous recombination. Constructs contained the Synechocystis sp. PCC 6803 $c p c B A(c p c)$ promoter to drive transgene expression, and a spectinomycin-resistance selectable marker (SmR). Arrows indicate the position of genomic DNA primers used to check for integration and complete chromosomal segregation in transformant lines. (B) Verification of compete chromosomal segregation for the introduced transgenes in YFP, LS, and BIS transformants by colony-PCR using the above-mentioned primers. Three independent transformant lines of LS (LS.1, LS.2, and LS.3) and BIS (BIS.1, BIS.2, and BIS.3) were verified. biphasic separation protocol described by Bligh and Dyer (1959). To determine if any accumulated terpenoids had partitioned to the media or adhered to the flask walls, the supernatant was returned to the original Erlenmeyer flask and a 5-mL dodecane overlay applied for $1 \mathrm{~h}$ for terpenoid extraction.

Aliquots of $200 \mu \mathrm{L}$ were harvested from the dodecane overlay at each experimental time point. These samples, and the chloroform extracts from the cell pellet, were analyzed directly using $1 \mu \mathrm{L}$ of sample injected into a GC-FID (Agilent 7890A) equipped with a DB-5-ms column (Agilent Technologies, Santa Clara, CA, USA; $30 \mathrm{~m} \times 0.25 \mathrm{~mm} ; 0.25 \mu \mathrm{m}$ film thickness) and operated using the temperature program: $60-310^{\circ} \mathrm{C}\left(\right.$ at $12^{\circ} \mathrm{C} \mathrm{min}{ }^{-1}$ ), and $\mathrm{H}_{2}$ carrier gas at $1.5 \mathrm{~mL} \mathrm{~min}^{-1}$. Quantification of limonene or $\alpha$-bisabolene from the dodecane or chloroform extracts was performed using standard curves constructed by GC peak integration of serial dilutions of limonene (Sigma-Aldrich, USA) and bisabolene (Alfa Aesar, USA) standards in the appropriate solvent. Due to the reverse solvent effect (Grob, 1983; Grob and Schilling, 1983), limonene was observed as a broad peak on the chromatogram when mixed with dodecane. Dilution of the sample with a solvent of lower molecular weight than the solute typically eliminates this problem. However, dilution pushed limonene below the limits of detection in some samples, and so limonene peaks were integrated using the area measured under the broad limonene peak. The bisabolene standard contained a number of discrete peaks, and $\sim 40 \%$ of the total combined peak areas were attributed to other sesquiterpenes with different retention times relative to $\alpha$-bisabolene. The weight contribution by $\alpha$ bisabolene was adjusted accordingly for the standard curves. Mass spectral analysis was conducted using a Varian 3800 GC and Varian 1200 quadrupole MS/MS equipped with a Rxi-5ms column (30 $\mathrm{m} \times 0.25 \mathrm{~mm} ; 0.25 \mu \mathrm{m}$ film thickness); temperature program: $60-310^{\circ} \mathrm{C}\left(\right.$ at $\left.12^{\circ} \mathrm{C} \mathrm{min}^{-1}\right)$; He carrier gas at $1.2 \mathrm{~mL} \mathrm{~min}^{-1}$; mass spectra, $70 \mathrm{eV}$, EI mode; ion source temperature $215^{\circ} \mathrm{C}$; scan mass range, $18-500$.

\section{EXCRETED ORGANIC ACID MEASUREMENTS}

Synechococcus cultures used for excreted metabolite assays were grown photoautotrophically in $50 \mathrm{~mL}$ batches with an inoculation density of $\mathrm{OD}_{730 \mathrm{~nm}}=2.0$. Cells were washed twice with $\mathrm{A}^{+}(-\mathrm{N})$ media to remove media nitrogen before inoculation in either nitrogen-replete or nitrogen-deplete media for experimentation. Aliquots of cell suspension $(1 \mathrm{~mL})$ were removed from the culture at appropriate time points and centrifuged to pellet cell biomass. The resulting supernatant was filtered through a $0.45-\mu \mathrm{m}$ silicon membrane and the filtrate used directly for highperformance liquid chromatography (HPLC) and nuclear magnetic resonance (NMR) spectroscopy. A Surveryor Plus (Thermo Scientific, Waltham MA) HPLC equipped with an Aminex fermentation monitoring column [(150 $\mathrm{mm} \times 7.8 \mathrm{~mm})$; Bio-Rad, Hercules, CA, USA] was used for HPLC analyses. $25 \mu \mathrm{L}$ of filtrate was injected for isocratic elution using $8 \mathrm{mM} \mathrm{H}_{2} \mathrm{SO}_{4}$ as the mobile phase with a flow rate of $0.5 \mathrm{~mL} \mathrm{~min}^{-1}$ and a column operating temperature of $45^{\circ} \mathrm{C}$. A refractive index (RI) detector operating at $50^{\circ} \mathrm{C}$ was used for metabolite identification and quantification. Metabolite peaks were integrated and quantified using standard curves constructed from serial dilutions of authentic standards 
(Sigma-Aldrich, USA). Proton NMR spectra were generated with a JEOL ECA $500 \mathrm{MHz}$ spectrometer; 128 scans were used with water-suppression.

\section{BIOMASS, CHLOROPHYLL a, CARBOHYDRATE, AND WHOLE CELL ABSORPTION SPECTRA MEASUREMENTS}

Cyanobacterial biomass accumulation was measured as dry cell weight (DCW), where $2 \mathrm{~mL}$ samples of culture were pelleted and washed with $8.25 \mathrm{mM}$ Tris- $\mathrm{HCl}(\mathrm{pH} 8.2)$ to remove any residual salt from the growth medium. Pellets were then resuspended in $2 \mathrm{~mL} 8.25 \mathrm{mM}$ Tris ( $\mathrm{pH} \mathrm{8.2)}$, and dried overnight at $80^{\circ} \mathrm{C}$ in pre-weighed aluminum dishes prior to measuring the DCW. The dry weight of $2 \mathrm{~mL} 8.25 \mathrm{mM}$ Tris ( $\mathrm{pH}$ 8.2) solution (without cells) was subtracted from the cell dry weight to eliminate Tris buffer contributions. Chlorophyll $a$ concentrations in cell suspensions were determined spectrophotometrically in 90\% methanol extracts according to Meeks and Castenholz (1971). Total reducing sugars (in glucose equivalents) were quantified by the anthrone assay, as described by Meuser et al. (2011), as a measure of the carbohydrate content of cells. For whole cell absorption spectra, cell suspensions were normalized to an $\mathrm{OD}_{730 \mathrm{~nm}}$ of 0.3 and measured spectrophotometrically $(400-800 \mathrm{~nm})$.

\section{RESULTS CONSTRUCTION OF SYNECHOCOCCUS sp. PCC 7002 STRAINS CARRYING MsLS AND AgBIS TRANSGENES}

A "NSI" region of the Synechococcus sp. PCC 7002 genome was identified between two open reading frames encoding hypothetical proteins and used as a site for transgene integration (described in Materials and Methods). The cpcBA promoter from Synechocystis sp. PCC 6803 (Xu et al., 2011) was used to drive expression of the TPS transgenes. Numerous lines were selected for each new strain generated to ensure that any phenotypes arising were a consistent feature of the specific transformation. Figure 2 shows the successful integration of the MsLS and AgBIS constructs at the NSI locus, as evidenced by the gel shift of the amplified colonyPCR product in transformant strains relative to the wild type. Integration of the YFP construct was also confirmed in the YFP transformant, which was designed as a control strain to identify phenotypes in the LS and BIS strains that may be attributed to chromosomal disruption at the NSI locus, rather than expression of the TPS transgenes. Importantly, the lack of a wild type-sized band in all transformant lines indicated that homoplasmy for the introduced transgenes was achieved.

\section{IDENTIFICATION OF LIMONENE AND $\alpha$-BISABOLENE AS END-PRODUCTS OF TRANSFORMANT STRAINS}

The use of an organic solvent overlay has proven to be a successful method for harvesting terpenoids from microbial cultures (Newman et al., 2006; Anthony et al., 2009; Alonso-Gutierrez et al., 2013; Bentley et al., 2013). GC-FID analyses of solvent overlays from LS (Figure 3A) and BIS (Figure 3B) transformants showed prominent peaks with similar retention times to commercial standards of L-limonene ( $4.40 \mathrm{~min}$ ) and bisabolene $(9.89 \mathrm{~min})$, respectively, which were absent from the wild type. Figure 4 shows the mass spectral analyses of putative L-limonene and $\alpha$-bisabolene peaks from transformant solvent extracts in comparison to the corresponding reference spectra from the NIST Mass Spectral Library (upper panels). The solvent also extracted additional products common to all strains, including wild type (Figure 3, 10.5 min retention time), which may be membrane lipids (accumulated levels were below detection limits for mass spectral analysis). A comparison of L-limonene and bisabolene commercially available standards with NIST reference spectra is also shown in Figure 4 (lower panels). The major peak $(9.89 \mathrm{~min})$ in the bisabolene standard (Figure 3B) was identified as $\alpha$-bisabolene, the peak at 9.94 min was bisabolol, while the small peaks with retention times $<9.98 \mathrm{~min}$ were all sesquiterpenes based on a mass to charge $(\mathrm{m} / \mathrm{z})$ of 204 .

\section{TRANSFORMANT FITNESS AND EFFECT OF DODECANE ON THE RATES OF PHOTOAUTOTROPHIC GROWTH}

Photoautotrophic growth rates, as measured by chlorophyll and biomass accumulation, were comparable between wild type and the YFP control strain (Figure $\mathbf{5 A}$, left panels). This verified that NSI is a suitable "neutral" site for transgene integration in Synechococcus sp. PCC 7002 under the defined experimental conditions, because cell growth rate was not adversely affected by the genetic manipulation at this locus. Rates of chlorophyll and biomass accumulation in the LS and BIS transformants were also comparable to that of the wild type (Figure 5, left panels), confirming that expression of the MsLS and AgBIS transgenes, and subsequent intracellular accumulation of limonene and $\alpha$-bisabolene, did not adversely affect cell fitness under our experimental conditions.

Dodecane was chosen as the solvent of choice for longerterm terpenoid extraction experiments due to its relatively low volatility, which enabled continuous extraction over multiple days (evaporative loss was determined as negligible over the five day cultivation period, data not shown). The $C_{12}$ chain length also allowed chromatographic separation from $\mathrm{C}_{10}$ limonene and $\mathrm{C}_{15}$ bisabolene. The rate of limonene and $\alpha$-bisabolene biosynthesis during the growth phase was measured over a 96-h time course using a $5 \%(\mathrm{v} / \mathrm{v})$ dodecane overlay. Notably, the presence of the dodecane overlay did not greatly affect chlorophyll content or biomass yield in any of the strains (Figure 5, right panels), indicating that atmospheric $\mathrm{CO}_{2}$ was able to efficiently diffuse though the dodecane layer and dissolve in the liquid media at concentrations that were not limiting for photoautotrophic growth under these conditions.

\section{LIMONENE AND BISABOLENE BIOSYNTHETIC RATES AND CELLULAR LOCALIZATION}

Three independent lines of the LS transformant consistently produced yields over $4 \mathrm{mg}$ limonene $\mathrm{L}_{\text {culture }}{ }^{-1}$, with the highest rate of $50 \mu \mathrm{g} \mathrm{L}$ culture ${ }^{-1} \mathrm{~h}^{-1}$ recorded over the exponential growth phase when cells were actively dividing (Figure 6A). As cultures reached higher cell densities, light limitation due to cell shading began to limit photosynthetic growth (Figure 5A), which likely translated into reduced rates of limonene biosynthesis. Assuming that carbon comprised $\sim 50 \%$ of the total biomass, the carbon partitioning to limonene was estimated at $0.3 \%$. Yields of $\alpha-$ bisabolene were considerably lower, where average yields in BIS transformant lines ranged between 0.5 and $0.7 \mathrm{mg} \alpha$-bisabolene $\mathrm{L}$ culture $^{-1}$ (Figure 6B). The highest $\alpha$-bisabolene production rate 


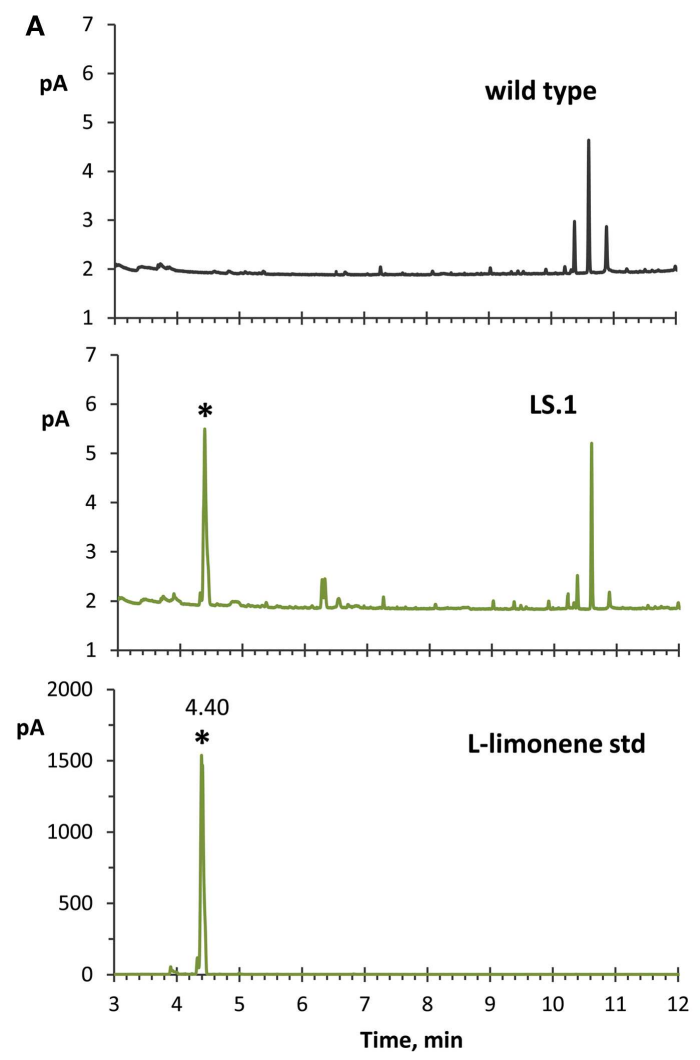

FIGURE 3 | GC-FID analyses of solvent overlay-extracted limonene and bisabolene from transformant strains. (A) Hexane-overlay extraction from the LS transformant (LS.1 line as a representative) showed accumulation of a product that was absent in the wild type, and with a similar retention time (4.40 min) to a commercial L-limonene standard. (B) Dodecane overlay extraction from the BIS transformant (BIS.1 line as a representative) revealed

was $6 \mu \mathrm{g} \mathrm{L}$ culture $\mathrm{C}^{-1} \mathrm{~h}^{-1}$ over the active growth phase, equivalent to $\sim 0.06 \%$ of assimilated carbon partitioning as $\alpha$-bisabolene.

A methanol:chloroform extraction was performed on cells harvested at the termination of the 96-h time course to assess whether any limonene or $\alpha$-bisabolene remained in the cells after the dodecane extraction procedure. On average, $11 \mu \mathrm{g}$ limonene $\mathrm{L}$ culture $^{-1}$ was extracted from LS cells (representing $0.25 \%$ of the total limonene yield), and $40 \mu \mathrm{g} \alpha$-bisabolene $\mathrm{L}$ culture ${ }^{-1}$ from BIS cells ( $8 \%$ of the total bisabolene yield) (Figure 6C). Therefore, almost 2.5-fold (mol:mol) more $\alpha$-bisabolene remained inside cells after dodecane extraction, compared to limonene. Relative to the overall terpenoid yields from LS and BIS transformants, a much greater fraction of $\alpha$-bisabolene was retained intracellularly compared to limonene. This may be explained, in part, by the mass difference between these two compounds, with the smaller limonene molecule having a greater rate of diffusion across the cell membrane.

Surprisingly, similar amounts of limonene and $\alpha$-bisabolene were extracted from harvested cells regardless of the presence or absence of the dodecane overlay (Figure 6C). Additional dodecane extractions of the spent media performed after cell harvesting did not reveal any terpenoids that partitioned to the media. The higher

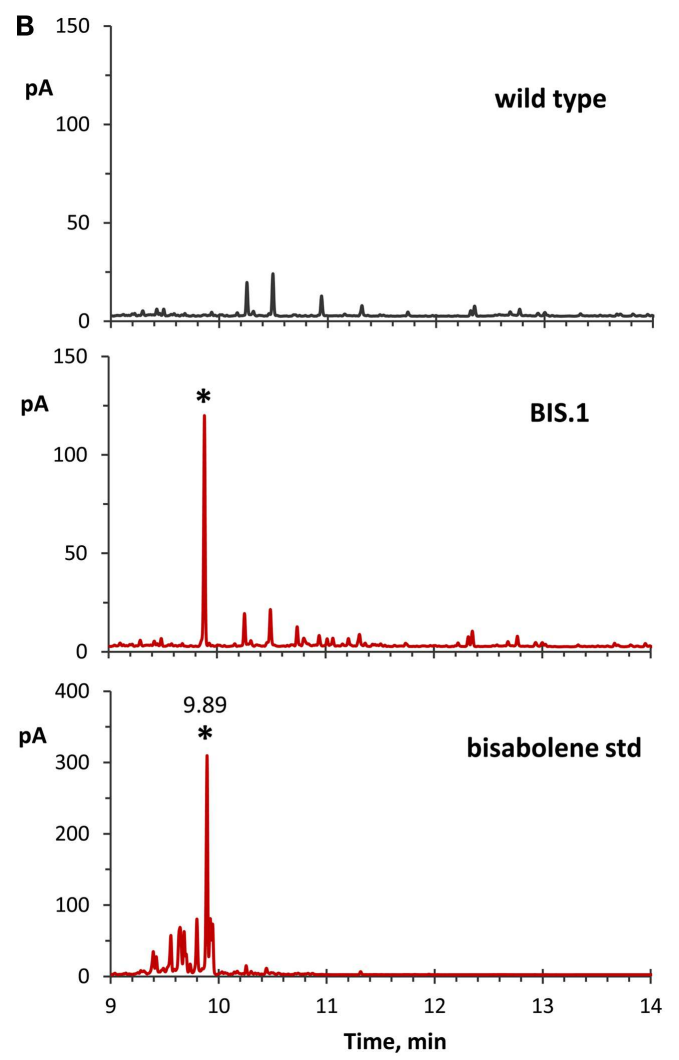

a peak with a similar retention time $(9.89 \mathrm{~min})$ to a bisabolene standard, which is absent in the wild type. For these experiments, dodecane was used for bisabolene extraction because of the extended period of time required to accumulate detectable levels of bisabolene, whereas hexane was applied for better limonene peak resolution due to the reverse solvent effect observed with dodecane.

overall product yields displayed by cultures overlayed with dodecane may be due to: (i) the trapping and solubilization of limonene and $\alpha$-bisabolene, which prevented evaporative loss, and/or (ii) the sequestration of limonene and $\alpha$-bisabolene from the cells, which alleviated a negative feedback inhibition mechanism.

\section{PHYSIOLOGICAL CHARACTERIZATION OF SYNECHOCOCCUS sp. PCC $7002 \Delta$ glgC}

At a light intensity of $250 \mu \mathrm{mol}$ photons $\mathrm{m}^{-2} \mathrm{~s}^{-1} \mathrm{PAR}$, the $\Delta g \operatorname{lgC}$ strain displayed slightly impaired photoautotrophic growth in nutrient-replete media, as measured by $\mathrm{OD}_{730 \mathrm{~nm}}$ and chlorophyll content (Figure 7A, left panels). In contrast to the wild type, $\Delta g l g C$ did not grow photoautotrophically in nitrogen-deplete medium (Figure 7A, right panels) due to its inability to degrade the lightharvesting phycobilisomes as a source of nitrogen (Carrieri et al., 2012; Grundel et al., 2012; Guerra et al., 2013; Hickman et al., 2013). Accordingly, the absorbance spectrum of $\Delta g l g C$ cells in nitrogen-deplete media shows the presence of phycobilin, which is absent in the wild type after nitrogen-deprivation (Figure 7B). As a result, the $\Delta g \lg C$ culture retained the blue-green hue under nitrogen-deprivation, while the wild type appeared more yellowgreen due to the unmasking of chlorophyll $a$ as the blue-pigmented 


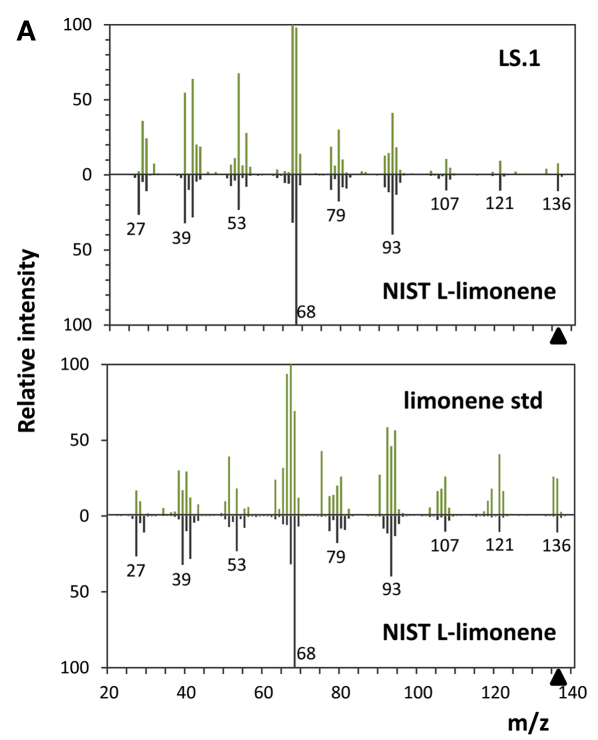

FIGURE 4 | Mass spectra analyses of limonene and $\alpha$-bisabolene. (A) Comparison of the L-limonene reference spectra from the NIST library with the spectra of the putative L-limonene peak in the LS.1 line (upper panel)

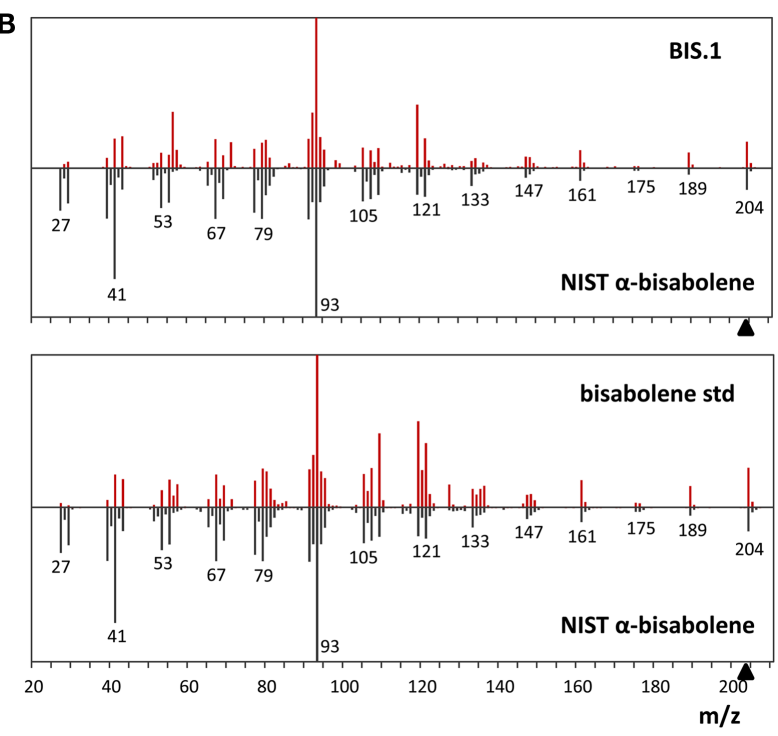

and the authentic L-limonene standard (lower panel). (B) $\alpha$-Bisabolene NIST reference spectra alignment with the putative $\alpha$-bisabolene peak in the BIS.1 line (upper panel) and a commercial bisabolene standard (lower panel). phycobiliproteins were degraded (Figure 7C). We confirmed that the reducing carbohydrate content of $\Delta g \lg C$ was diminished compared to the wild type, particularly during stationary phase at $48 \mathrm{~h}$ in nutrient-replete media, and also during the first $24 \mathrm{~h}$ after the onset of nitrogen-deprivation (Figure 7D). These data confirmed that $\Delta g l g C$ is defective in glycogen biosynthesis, and that our experimental conditions replicated the phenotypes previously observed in $\Delta g \operatorname{lgC}$ mutants of other cyanobacterial species (Carrieri et al., 2012; Grundel et al., 2012; Guerra et al., 2013; Hickman et al., 2013), and in Synechococcus sp. PCC 7002 (Guerra et al., 2013).

\section{ORGANIC ACID EXCRETION IN $\Delta g I g C$ AND ITS EFFECT ON TERPENOID YIELDS}

Consistent with studies in Synechocystis sp. PCC 6803 (Carrieri et al., 2012; Grundel et al., 2012) and Synechococcus elongatus PCC 7942 (Hickman et al., 2013), we identified pyruvate, $\alpha$-ketoglutarate, and succinate in the spent media of nitrogendeprived Synechococcus sp. PCC $7002 \Delta g \operatorname{lgC}$ cultures using HPLC analysis (Figure 8A, left panels), through a comparison of elution times with known standards (Figure 8B). However, we also detected $\alpha$-ketoisocaproate and acetate within the same concentration range, metabolites which were not previously reported in the $\Delta g \lg C$ strains of the other cyanobacterial species. Acetate is a common fermentation product putatively derived from acetylCoA in cyanobacterial metabolism (Xu et al., 2013), while $\alpha$ ketoisocaproate is the immediate precursor to leucine. This highlights subtle metabolic distinctions in the metabolism of the $\Delta g \operatorname{lgC}$ mutants between the model cyanobacterial species. An additional unique observation was the excretion of the same organic acids by $\Delta g l g C$ in nutrient-replete media at the onset of stationary phase, when wild type cells would normally begin to increase glycogen stores for energy reserves (Figure 8A, right panels). However, on a per-cell basis, these concentrations were much lower relative to the concentrations of those excreted during nitrogen starvation. NMR was used as a second, independent method to verify the identity of these metabolites (Figure 8C). Pyruvate, succinate, and $\alpha$ ketoisocaproate accumulated at concentrations of $\sim 250 \mu \mathrm{M}$, while $\alpha$-ketoglutarate accumulated at $\sim 200 \mu \mathrm{M}$, and acetate $\sim 500 \mu \mathrm{M}$ under nitrogen starvation.

To assess whether the metabolic flux imbalance in $\Delta g \operatorname{lgC}$ redirected metabolites toward terpenoid biosynthesis, we grew the $\Delta g \lg C$ :LS and $\Delta g \operatorname{lgC}$ :BIS transformants under conditions of nitrogen starvation. The total yields of limonene and $\alpha$-bisabolene were extremely low relative to the culture volume, which was not surprising given the absence of growth during nitrogen starvation (data not shown). However, terpenoid yield relative to biomass was relatively constant, regardless of whether cultures were grown in the presence or absence of nitrogen. In fact, throughout this study, terpenoid yield as a function of biomass remained relatively constant among all combinations of genetic background $( \pm g \lg C)$, nutrient availability ( \pm nitrogen), and growth phase (exponential vs. stationary). This indicates that terpenoid biosynthesis is coupled to cell growth and that flux through the terpenoid pathway is tightly regulated, even in the face of metabolic perturbations that increase feedstock availability.

Interestingly, however, we observed a higher extracellular concentration of excreted organic acids in the limonene-producing $\triangle g l g C:$ LS strain, relative to the $\Delta g \lg C$ and $\Delta g \lg C$ :BIS strains (Figure 9). Under nitrogen starvation, $\Delta g l g C$ :LS accumulated $\sim 2.5$-fold more $\alpha$-ketoglutarate, and almost twofold more $\alpha$ ketoisocaproate, pyruvate, and succinate in the spent media compared with the $\Delta g \operatorname{lgC}$ strain, despite no detectable photoautotrophic growth in either strains. It is unclear whether the higher 


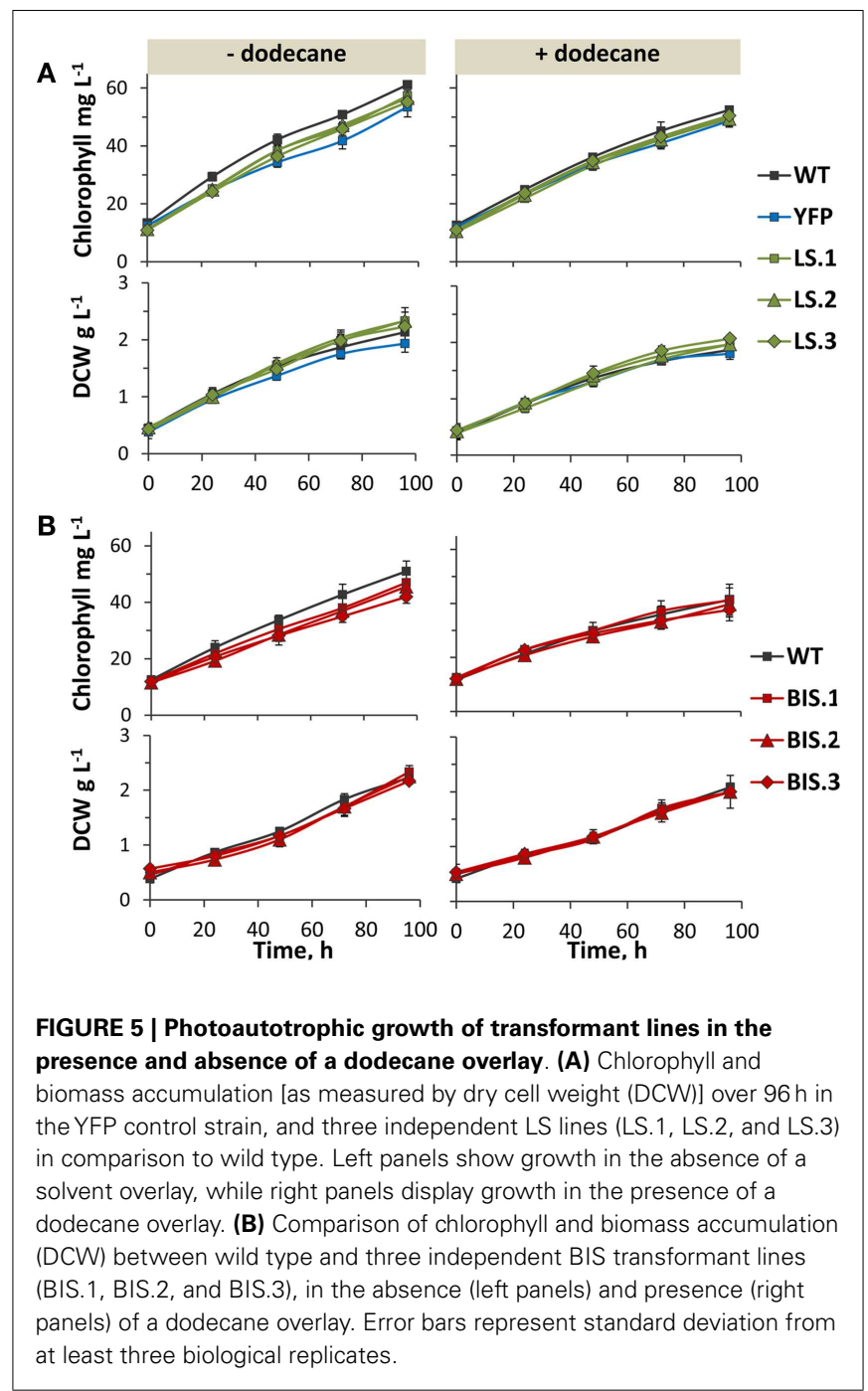

concentrations in the $\Delta g l g C$ :LS strain are due to increased metabolism toward these end-products, or if a greater proportion of the organic acids are excreted from the cell, but provides an interesting line of future investigation.

\section{DISCUSSION}

We have demonstrated for the first time, the synthesis of limonene in Synechococcus sp. PCC 7002, and the first synthesis of $\alpha$ bisabolene in a photosynthetic microorganism. The choice of $M$. spicata (-)-4S-limonene synthase and A. grandis (E)- $\alpha-$ bisabolene synthase as representative monoterpene and sesquiterpene synthases was based on proven successes in heterotrophic microbial production hosts (Carter et al., 2003; Peralta-Yahya et al., 2011; Alonso-Gutierrez et al., 2013; Ozaydin et al., 2013).

Importantly, (-)-4S-limonene synthase and $(E)$ - $\alpha$-bisabolene synthase are known to have high product specificity (Alonso et al., 1992; Rajaonarivony et al., 1992; Bohlmann et al., 1998), which is unusual for TPSs. Most TPSs generate multiple products from the prenyl diphosphate substrate as a result of the highly unstable carbocationic intermediates generated during the
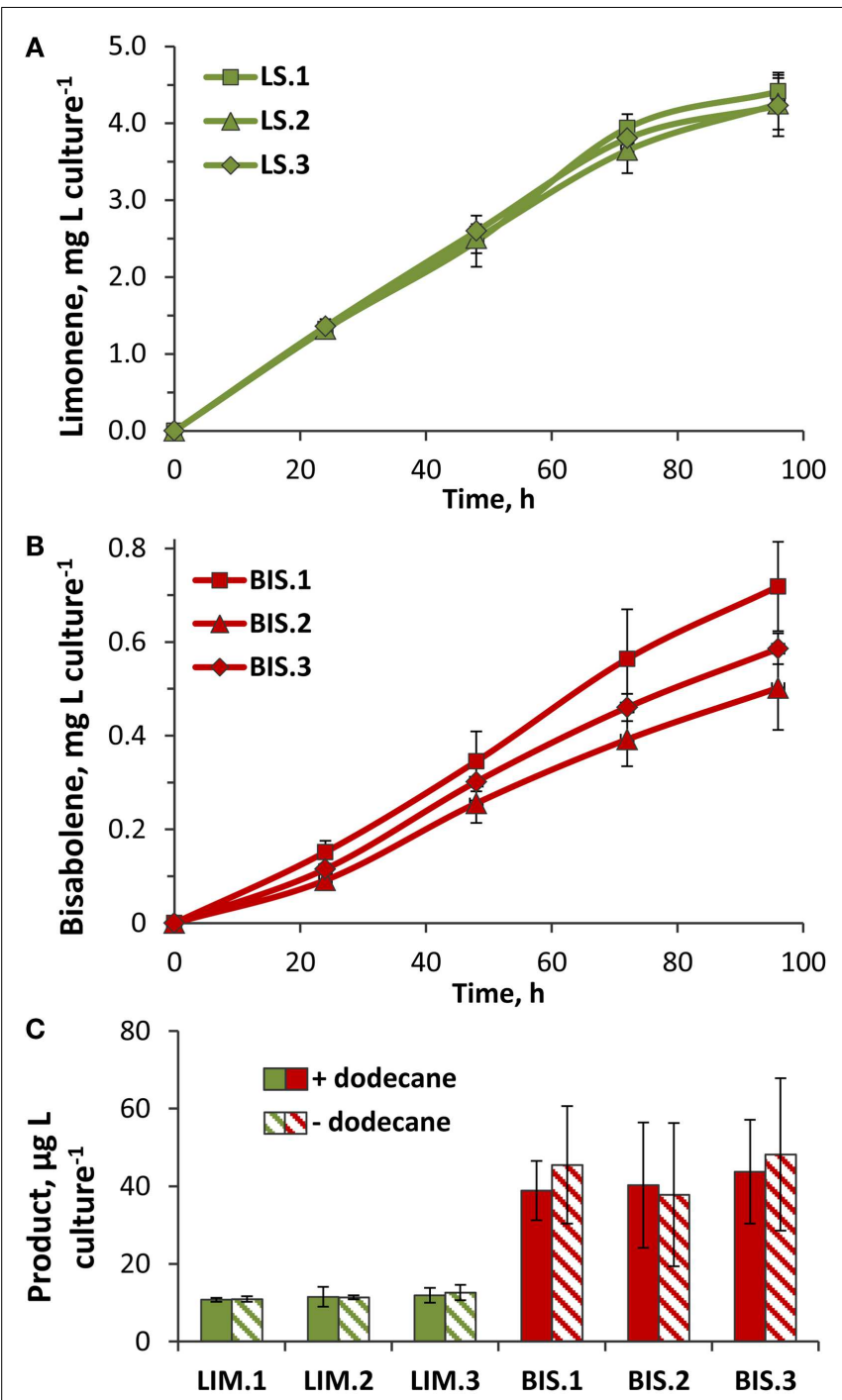

FIGURE 6 | Rates of limonene and $\alpha$-bisabolene production in transformant lines. (A) Limonene accumulation in three independent LS transformant lines (LS.1, LS.2, and LS.3). Yields were quantified by GC-FID analysis of limonene extracted by a dodecane overlay during the 96-h growth phase (Figure 5A, +dodecane). (B) $\alpha$-Bisabolene extracted from three independent BIS lines (BIS.1, BIS.2, and BIS.3) over the 96-h growth phase (Figure 5B, +dodecane) using a dodecane overlay. (C) Yields of limonene and $\alpha$-bisabolene extracted from cells harvested after $96 \mathrm{~h}$ of photoautotrophic growth (Figure 5) in the presence (solid bars) or absence (hatched bars) of a dodecane overlay. Terpenoids were extracted from cells using a methanol:chloroform extraction method, and quantified by GC-FID. Error bars represent standard deviation from at least three biological replicates.

electrophilic reaction. Accordingly, these enzymes also produced pure $\mathrm{L}$-limonene and $\alpha$-bisabolene products in Synechococcus sp. PCC 7002; however, the yield of L-limonene was much greater than that of $\alpha$-bisabolene. This could be explained by the superior enzyme kinetics of the M. spicata $\mathrm{L}$-limonene synthase, which has a $K_{\mathrm{m}}(\mathrm{GPP})$ of $1.8 \mu \mathrm{M}$ and $k_{\text {cat }}$ of $0.3 \mathrm{~s}^{-1}$ (Alonso et al., 1992; Rajaonarivony et al., 1992), compared to the A. grandis 


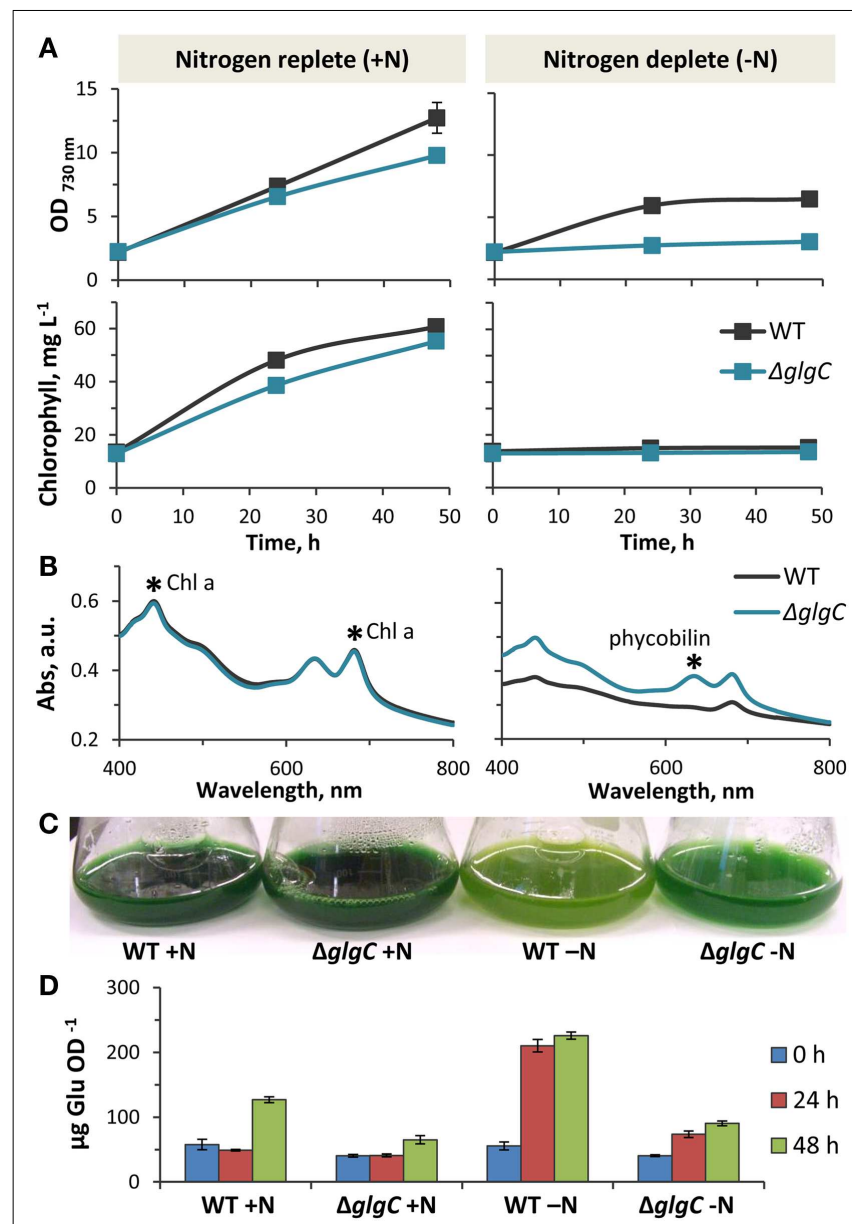

FIGURE 7 | Physiology of Synechococcus sp. PCC 7002 sglgC.

(A). Comparison of photoautotrophic growth rates in wild type and $\Delta g / g C$ cultures in nitrogen-replete and nitrogen-deplete media, as measured by changes of optical density $(730 \mathrm{~nm})$ and chlorophyll content over $48 \mathrm{~h}$. (B) Whole cell absorption spectra of wild type and $\Delta \mathrm{glgC}$ cells after $48 \mathrm{~h}$ growth in nitrogen-replete and nitrogen-deplete media. (C) Differences in pigmentation of wild type and $\Delta g l g C$ cultures after $48 \mathrm{~h}$ growth in the presence or absence of nitrogen in the media. (D) Carbohydrate content (reported as glucose equivalents) of wild type and $\Delta \mathrm{g} / \mathrm{gC}$ cells at the different stages of growth $(0,24$, and $48 \mathrm{~h})$ shown in (A). Error bars represent standard deviation from three biological replicates, and are hidden beneath the marker if not apparent.

(E)- $\alpha$-bisabolene synthase, with a high $K_{\mathrm{m}}$ (FPP) of $49.5 \mu \mathrm{M}$ and very low $k_{\text {cat }}$ of $0.11 \mathrm{~min}^{-1}$ (McAndrew et al., 2011). Substrate availability is also likely to play a significant role because monoterpenes and sesquiterpenes are absent in most cyanobacteria, and so GPP and FPP substrates may not accumulate to the levels seen in plants. However, GPP must accumulate in significant quantities as the precursor to GGPP, from which longer chained cyanobacterial terpenoids are synthesized (e.g., carotenoids), and so may also explain the greater L-limonene yield relative to $\alpha$-bisabolene.

The cyanobacterium Synechocystis sp. PCC 6803 has previously been engineered to produce $\beta$-phellandrene monoterpenes (Bentley et al., 2013) and $\beta$-caryophyllene sesquiterpenes
(Reinsvold et al., 2011). The yield of $\beta$-phellandrene was $133 \mu \mathrm{gDCW}^{-1}$, as measured by a non-continuous heptaneoverlay extraction method. Intracellular titers of $\beta$-caryophyllene were $\sim 4 \mu \mathrm{g} \mathrm{gDCW}^{-1}$, as measured by a methanol:choloroform extraction method. However, these yields may have been underestimated due to the volatile nature of the terpenoid products. Evaporation was attributed to major losses of the amorphadiene sesquiterpene from $E$. coli liquid cultures, and a dodecane overlay was demonstrated to reduce the loss (Newman et al., 2006). Recently, the nitrogen-fixing cyanobacterium Anabaena sp. PCC 7120 was engineered for limonene production via heterologous expression of the Picea sitchensis limonene synthase (Halfmann et al., 2014). The total yield of limonene $\left(114 \mu \mathrm{g} \mathrm{L}^{-1}\right)$ was found to partition as a volatile gas in the culture headspace, and titers were improved $\sim$ ninefold upon overexpression of three rate-limiting enzymes involved in terpenoid metabolism. Here, we report the yields of $1.7 \mathrm{mg} \mathrm{gDCW}^{-1}\left(4 \mathrm{mg} \mathrm{L}^{-1}\right)$ of limonene and $0.3 \mathrm{mg} \mathrm{gDCW}^{-1}\left(0.6 \mathrm{mg} \mathrm{L}^{-1}\right)$ of $\alpha$-bisabolene in Synechococcus sp. PCC 7002, upon expression of the single TPS genes, and using a continuous dodecane overlay extraction method that may reduce product loss due to evaporation.

It is also conceivable that dodecane may actively sequester terpenoids from the cell, thereby relieving any negative feedback pressures exerted by the products thus promoting higher endproduct yields. Indeed, a 20\% (v/v) dodecane overlay was found to alleviate growth inhibition of Synechocystis sp. PCC 6803 that was induced by the addition of commercial farnesene at concentrations as low as $0.04 \%$ (v/v) (Hellier et al., 2013). Further support for this theory was demonstrated in E. coli, where heterologous expression of an export pump in a limonene-producing strain increased limonene yield by $\sim 60 \%$ (Dunlop et al., 2011). The microbial toxicity of bisabolene appears to be very low, with concentrations of commercial bisabolene up to $20 \%$ (v/v) exerting no effect on cell growth in E. coli and yeast (Peralta-Yahya et al., 2011). Although toxicity information is useful in this respect, the product feedback inhibition may not always manifest as growth inhibition. To fully understand the increased limonene and $\alpha$ bisabolene yields in the presence of dodecane, investigations are required: (i) to probe terpenoid (or other) pathway inhibition by these end-products as well as (ii) to quantify the biologically produced limonene and bisabolene lost via evaporation in the absence of dodecane. It is important to establish whether the product naturally separates from biomass and how the physical properties of the product (mass, boiling point) influence this, or whether the product exerts feedback inhibition so that engineering efforts may be targeted toward this.

Targeted metabolic flux redistribution among the major carbon sinks is the key to producing novel end-products from engineered microbes at commercially available quantities. The goal remains to increase product yield at the expense of biomass accumulation (cell growth and division), without negatively impacting metabolisms associated with the assimilation and direction of carbon to the product. Although disruption of glycogen biosynthesis in Synechococcus sp. PCC 7002 produced an oversupply of central metabolites ( $\alpha$-ketoisocaproate, $\alpha$-ketoglutarate, pyruvate, succinate, and acetate) (Figure 10), there was no apparent increase in flux though the MEP terpenoid pathway as conveyed by yields 

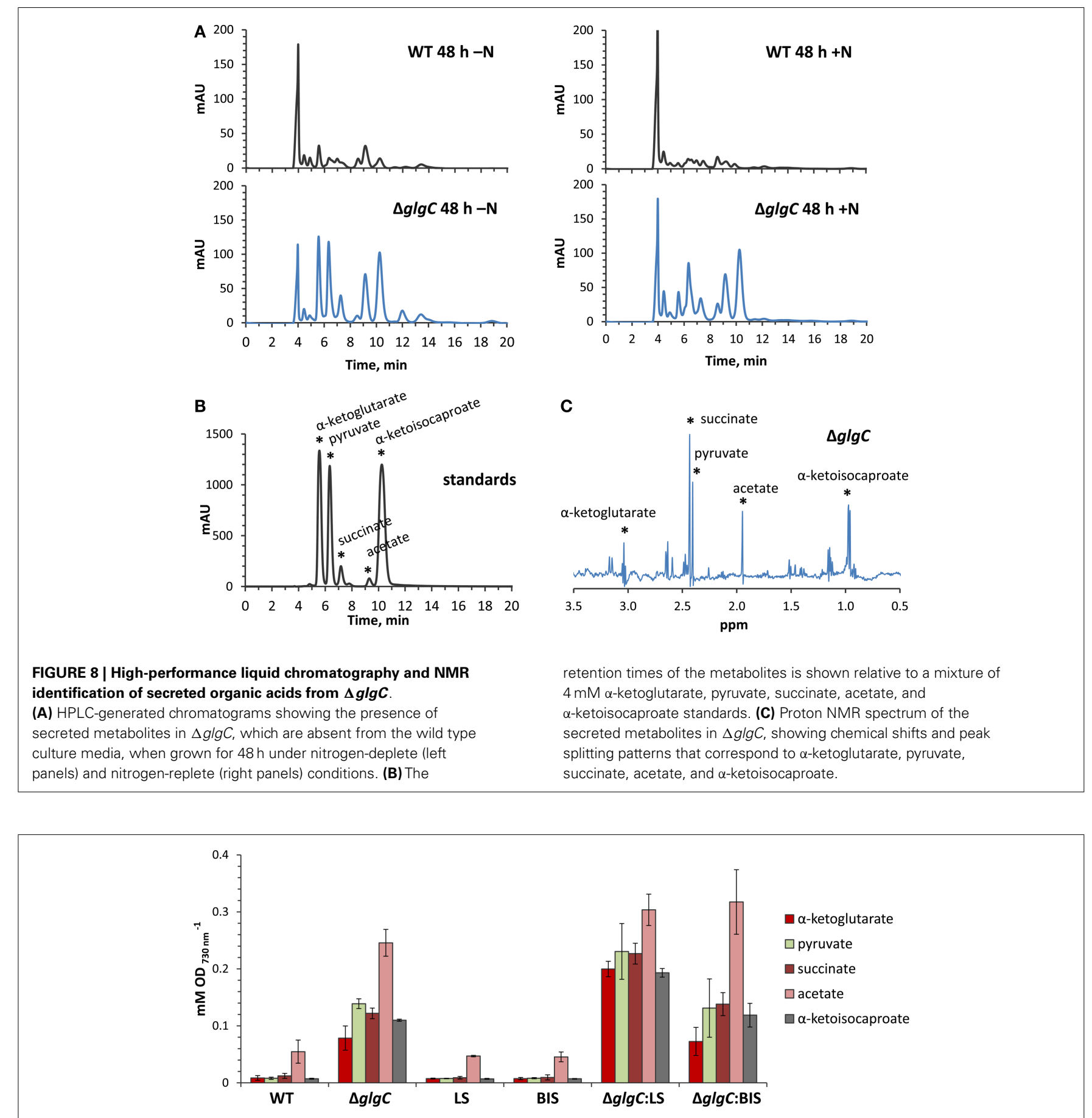

FIGURE 9 | Accumulation of excreted organic acids in the spend media of cells grown under nitrogen starvation for $\mathbf{4 8} \mathbf{h}$. Concentrations of organic acids were measured by HPLC analysis and presented relative to cell optical density at $730 \mathrm{~nm}$. Error bars represent standard deviation from three biological replicates.

of limonene and $\alpha$-bisabolene reporter products. Furthermore, such a severe perturbation of central carbon metabolism had a detrimental effect on the organism fitness as manifested by a complete growth inhibition. To that end, introduction of alternative routes for carbon partitioning have proven successful in cyanobacteria as carbon fixation rates are stimulated by providing alternative sinks for photosynthetically derived NADPH and ATP (Ducat et al., 2012; Li et al., 2014). While the MEP terpenoid pathway utilizes both NADPH and ATP as cofactors, growth and pathway optimization are required so that photosynthesis 


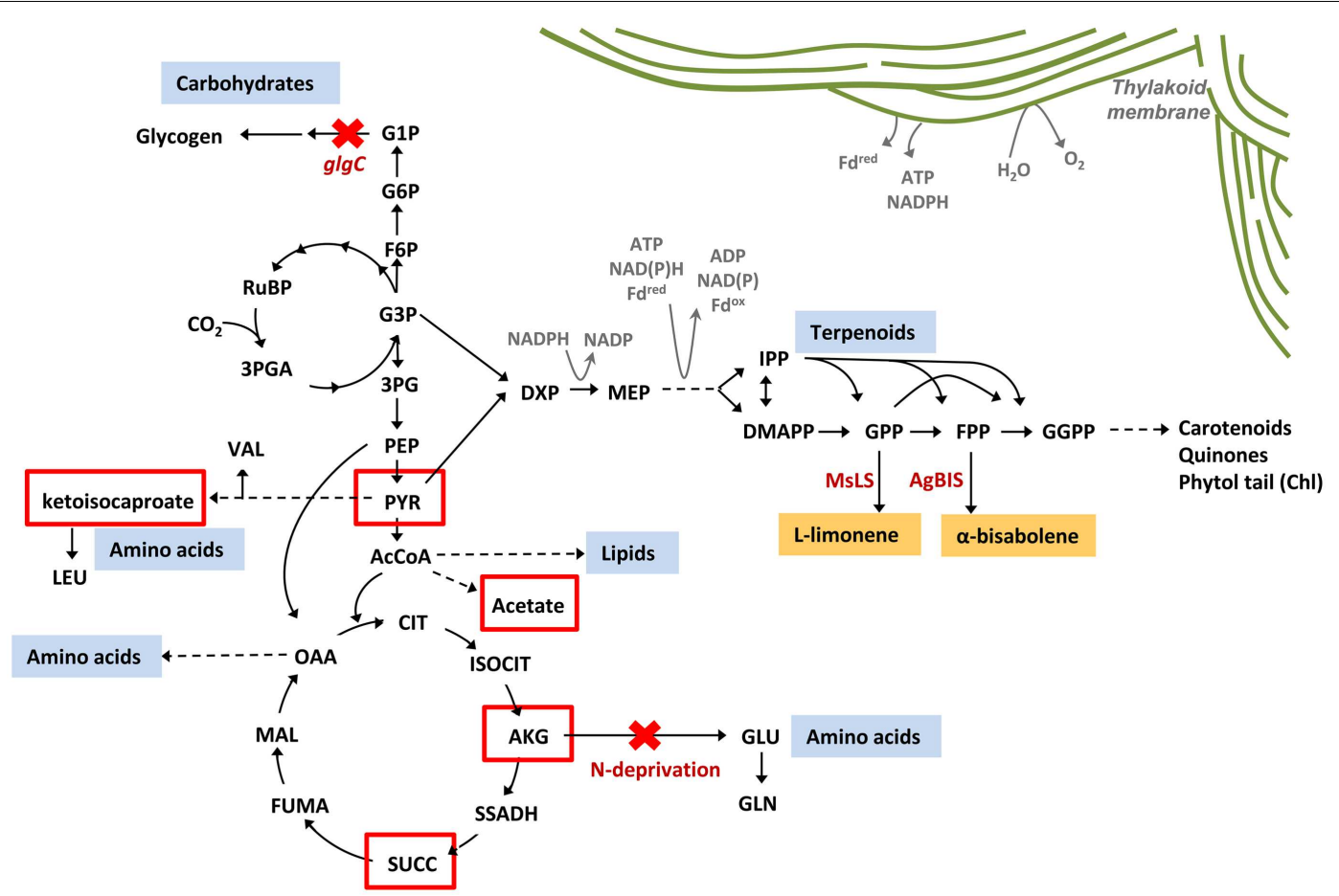

FIGURE 10 | Metabolic redistribution in Synechococcus sp. PCC 7002 upon simultaneous blocks to protein and carbohydrate sinks. Protein (amino acids), carbohydrates (mainly glycogen), and lipids represent the major metabolic sinks in Synechococcus sp. PCC 7002. When metabolic flux to glycogen and amino acids were simultaneously blocked, through inactivation of $\Delta g l g C$ and elimination of nitrogen from the culture medium (red crosses), a suite of organic acids were secreted from the cell as overflow metabolites from central metabolism (red boxes). There is potential to redirect these spill-over intermediates toward biotechnologically relevant pathways to increase yields of engineered products, such as limonene and $\alpha$-bisabolene via the terpenoid biosynthetic pathway. and terpenoid biosynthesis remain active despite major metabolic redistributions.

\section{ACKNOWLEDGMENTS}

We would like to thank D. A. Bryant for providing the Synechococcus sp. PCC $7002 \Delta g \operatorname{lgC}$ strain and the pAQ1 plasmid containing the $c p c B A$ promoter. This research was supported by the Genomic Science Program (GSP), Office of Biological and Environmental Research (OBER), U.S. Department of Energy, and is a contribution of the PNNL Biofuels Scientific Focus Area (Alexander S. Beliaev and Matthew C. Posewitz). Additional resources for this project were provided by the Air Force Office of Scientific Research under Grant FA9550-11-1-0211 (Matthew C. Posewitz).

\section{REFERENCES}

Alonso, W. R., Rajaonarivony, J. I. M., Gershenzon, J., and Croteau, R. (1992). Purification of $4 S$-limonene synthase, a monoterpene cyclase from the glandular trichomes of peppermint (Mentha x piperita) and spearmint (Mentha spicata). J. Biol. Chem. 267, 7582-7587.

Alonso-Gutierrez, J., Chan, R., Batth, T. S., Adams, P. D., Keasling, J. D., Petzold, C. J., et al. (2013). Metabolic engineering of Escherichia coli for limonene and perillyl alcohol production. Metab. Eng. 19, 33-41. doi:10.1016/j.ymben.2013.05.004

Anthony, J. R., Anthony, L. C., Nowroozi, F., Kwon, G., Newman, J. D., and Keasling, J. D. (2009). Optimization of the mevalonate-based isoprenoid biosynthetic pathway in Escherichia coli for production of the anti-malarial drug precursor amorpha-4,11-diene. Metab. Eng. 11, 13-19. doi:10.1016/j.ymben.2008.07.007
Atsumi, S., Higashide, W., and Liao, J. C. (2009). Direct photosynthetic recycling of carbon dioxide to isobutyraldehyde. Nat. Biotechnol. 27, 1177-1180. doi:10.1038/nbt.1586

Bentley, F. K., García-Cerdán, J. G., and Chen, H.-C. (2013). Paradigm of monoterpene ( $\beta$-phellandrene) hydrocarbons production via photosynthesis in cyanobacteria. Bioenerg. Res. 6, 917-929. doi:10.1007/s12155-013-9325-4

Bentley, F. K., and Melis, A. (2012). Diffusion-based process for carbon dioxide uptake and isoprene emission in gaseous/aqueous two-phase photobioreactors by photosynthetic microorganisms. Biotechnol. Bioeng. 109, 100-109. doi:10.1002/bit.23298

Bentley, F. K., Zurbriggen, A., and Melis, A. (2014). Heterologous expression of the mevalonic acid pathway in cyanobacteria enhances endogenous carbon partitioning to isoprene. Mol. Plant 7, 71-86. doi:10.1093/mp/sst134

Bligh, E. G., and Dyer, W. J. (1959). A rapid method of total lipid extraction and purification. Can. J. Biochem. Physiol. 37, 911-917. doi:10.1139/o59-099

Bohlmann, J., Crock, J., Jetter, R., and Croteau, R. (1998). Terpenoid-based defenses in conifers: cDNA cloning, characterization, and functional expression of wound-inducible (E)-alpha-bisabolene synthase from grand fir (Abies grandis). Proc. Natl. Acad. Sci. U.S.A. 95, 6756-6761. doi:10.1073/pnas.95.12.6756

Carrieri, D., Paddock, T., Maness, P. C., Seibert, M., and Yu, J. P. (2012). Photocatalytic conversion of carbon dioxide to organic acids by a recombinant cyanobacterium incapable of glycogen storage. Energy Environ. Sci. 5, 9457-9461. doi:10.1039/c2ee23181f

Carter, O. A., Peters, R. J., and Croteau, R. (2003). Monoterpene biosynthesis pathway construction in Escherichia coli. Phytochemistry 64, 425-433. doi:10.1016/ S0031-9422(03)00204-8

Colby, S. M., Alonso, W. R., Katahira, E. J., Mcgarvey, D. J., and Croteau, R. (1993). $4 S$-limonene synthase from the oil glands of spearmint (Mentha spicata). cDNA isolation, characterization, and bacterial expression of the catalytically active monoterpene cyclase. J Biol Chem 268, 23016-23024. 
Davies, F. K., Jinkerson, R. E., and Posewitz, M. C. (2014). Toward a photosynthetic microbial platform for terpenoid engineering. Photosyn. Res. doi:10.1007/ s11120-014-9979-6

Ducat, D. C., Avelar-Rivas, J. A., Way, J. C., and Silver, P. A. (2012). Rerouting carbon flux to enhance photosynthetic productivity. Appl. Environ. Microbiol. 78, 2660-2668. doi:10.1128/AEM.07901-11

Duetz, W. A., Bouwmeester, H., Van Beilen, J. B., and Witholt, B. (2003). Biotransformation of limonene by bacteria, fungi, yeasts, and plants. Appl. Microbiol. Biotechnol. 61, 269-277. doi:10.1007/s00253-003-1221-y

Dunlop, M. J., Dossani, Z. Y., Szmidt, H. L., Chu, H. C., Lee, T. S., Keasling, J. D., et al. (2011). Engineering microbial biofuel tolerance and export using efflux pumps. Mol. Syst. Biol. 7, 487-493. doi:10.1038/msb.2011.21

Eaton-Rye, J. J. (2011). Construction of gene interruptions and gene deletions in the cyanobacterium Synechocystis sp. strain PCC 6803. Methods Mol. Biol. 684, 295-312. doi:10.1007/978-1-60761-925-3_22

Grob, K. (1983). Broadening of peaks eluted before the solvent in capillary GC. Part 1: the role of solvent trapping. Chromatographia 17, 357-360. doi:10.1007/ BF02262373

Grob, K., and Schilling, B. (1983). Broadening of peaks eluted before the solvent in capillary GC. Part II: the role of phase soaking. Chromatographia 17, 361-367. doi:10.1007/BF02262373

Grundel, M., Scheunemann, R., Lockau, W., and Zilliges, Y. (2012). Impaired glycogen synthesis causes metabolic overflow reactions and affects stress responses in the cyanobacterium Synechocystis sp. PCC 6803. Microbiology 158, 3032-3043. doi:10.1099/mic.0.062950-0

Guerra, L. T., Xu, Y., Bennette, N., Mcneely, K., Bryant, D. A., and Dismukes, G. C. (2013). Natural osmolytes are much less effective substrates than glycogen for catabolic energy production in the marine cyanobacterium Synechococcus sp. strain PCC 7002. J. Biotechnol. 166, 65-75. doi:10.1016/j.jbiotec.2013.04.005

Halfmann, C., Gu, L., and Zhou, R. (2014). Engineering cyanobacteria for production of a cyclic hydrocarbon from $\mathrm{CO}_{2}$ and $\mathrm{H}_{2} \mathrm{O}$. Green Chem 16, 3175-3185. doi:10.1039/C3GC42591F

Hellier, P., Al-Haj, L., Talibi, M., Purton, S., and Ladommatos, N. (2013). Combustion and emissions characterization of terpenes with a view to their biological production in cyanobacteria. Fuel 111, 670-688. doi:10.1016/j.fuel.2013.04.042

Hickman, J. W., Kotovic, K. M., Miller, C., Warrener, P., Kaiser, B., Jurista, T., et al. (2013). Glycogen synthesis is a required component of the nitrogen stress response in Synechococcus elongatus PCC 7942. Algal Res. 2, 98-106. doi:10.1016/j.algal.2013.01.008

Li, X., Shen, C. R., and Liao, J. C. (2014). Isobutanol production as an alternative metabolic sink to rescue the growth deficiency of the glycogen mutant of Synechococcus elongatus PCC 7942. Photosyn. Res. 120, 301-310. doi:10.1007/s11120014-9987-6

Lindberg, P., Park, S., and Melis, A. (2010). Engineering a platform for photosynthetic isoprene production in cyanobacteria, using Synechocystis as the model organism. Metab. Eng. 12, 70-79. doi:10.1016/j.ymben.2009.10.001

McAndrew, R. P., Peralta-Yahya, P. P., Degiovanni, A., Pereira, J. H., Hadi, M. Z., Keasling, J. D., et al. (2011). Structure of a three-domain sesquiterpene synthase: a prospective target for advanced biofuels production. Structure 19, 1876-1884. doi:10.1016/j.str.2011.09.013

Meeks, J., and Castenholz, R. (1971). Growth and photosynthesis in an extreme thermophile, Synechococcus lividus (cyanophyta). Arch. Mikrobiol. 78, 25-41. doi:10.1007/BF00409086

Meuser, J. E., Boyd, E. S., Ananyev, G., Karns, D., Radakovits, R., Murthy, U. M. N., et al. (2011). Evolutionary significance of an algal gene encoding an FeFehydrogenase with F-domain homology and hydrogenase activity in Chlorella variabilis NC64A. Planta 234, 829-843. doi:10.1007/s00425-011-1431-y

Newman, J. D., Marshall, J., Chang, M., Nowroozi, F., Paradise, E., Pitera, D., et al. (2006). High-level production of amorpha-4,11-diene in a two-phase partitioning bioreactor of metabolically engineered Escherichia coli. Biotechnol. Bioeng. 95, 684-691. doi:10.1002/bit.21017
Oliver, J. W. K., Machado, I. M. P., Yoneda, H., and Atsumi, S. (2013). Cyanobacterial conversion of carbon dioxide to 2,3-butanediol. Proc. Natl. Acad. Sci. U.S.A. 110, 1249-1254. doi:10.1073/pnas.1213024110

Oliver, J. W. K., Machado, I. M. P., Yoneda, H., and Atsumi, S. (2014). Combinatorial optimization of cyanobacterial 2,3-butanediol production. Metab. Eng. 22, 76-82. doi:10.1016/j.ymben.2014.01.001

Ozaydin, B., Burd, H., Lee, T. S., and Keasling, J. D. (2013). Carotenoid-based phenotypic screen of the yeast deletion collection reveals new genes with roles in isoprenoid production. Metab. Eng. 15, 174-183. doi:10.1016/j.ymben.2012. 07.010

Peralta-Yahya, P. P., Ouellet, M., Chan, R., Mukhopadhyay, A., Keasling, J. D., and Lee, T. S. (2011). Identification and microbial production of a terpene-based advanced biofuel. Nat. Commun. 2, 483. doi:10.1038/ncomms1494

Rajaonarivony, J. I. M., Gershenzon, J., and Croteau, R. (1992). Characterization and mechanism of (4S)-limonene synthase, a monoterpene cyclase from the glandular trichomes of peppermint (Mentha X piperita). Arch. Biochem. Biophys. 296, 49-57. doi:10.1016/0003-9861(92)90543-6

Reinsvold, R. E., Jinkerson, R. E., Radakovits, R., Posewitz, M. C., and Basu, C. (2011). The production of the sesquiterpene $\beta$-caryophyllene in a transgenic strain of the cyanobacterium Synechocystis. J. Plant Physiol. 168, 848-852. doi:10.1016/j.jplph.2010.11.006

Stevens, S. E. J., Patterson, C. O. P., and Myers, J. (1973). The production of hydrogen peroxide by blue-green algae: a survey. J. Phycol. 9, 427-430. doi:10.1111/j.0022-3646.1973.00427.x

Trapp, S. C., and Croteau, R. B. (2001). Genomic organization of plant terpene synthases and molecular evolutionary implications. Genetics 158, 811-832.

Ungerer, J., Tao, L., Davis, M., Ghirardi, M., Maness, P. C., and Yu, J. P. (2012). Sustained photosynthetic conversion of $\mathrm{CO}_{2}$ to ethylene in recombinant cyanobacterium Synechocystis 6803. Energy Environ. Sci. 5, 8998-9006. doi:10.1039/ c2ee22555g

Williams, D. C., McGarvey, D. J., Katahira, E. J., and Croteau, R. (1998). Truncation of limonene synthase preprotein provides a fully active "pseudomature" form of this monoterpene cyclase and reveals the function of the amino-terminal arginine pair. Biochemistry 37, 12213-12220. doi:10.1021/bi980854k

Xu, Y., Alvey, R. M., Byrne, P. O., Graham, J. E., Shen, G., and Bryant, D. A. (2011). Expression of genes in cyanobacteria: adaptation of endogenous plasmids as platforms for high level gene expression in Synechococcus sp. PCC 7002. Methods Mol. Biol.684, 273-293. doi:10.1007/978-1-60761-925-3_21

Xu, Y., Guerra, L. T., Li, Z., Ludwig, M., Dismukes, G. C., and Bryant, D. A. (2013) Altered carbohydrate metabolism in glycogen synthase mutants of Synechococcus sp. strain PCC 7002: cell factories for soluble sugars. Metab. Eng. 16, 56-67. doi:10.1016/j.ymben.2012.12.002

Conflict of Interest Statement: The authors declare that the research was conducted in the absence of any commercial or financial relationships that could be construed as a potential conflict of interest.

Received: 09 April 2014; accepted: 04 June 2014; published online: 19 June 2014. Citation: Davies FK, Work VH, Beliaev AS and Posewitz MC (2014) Engineering limonene and bisabolene production in wild type and a glycogen-deficient mutant of Synechococcus sp. PCC 7002. Front. Bioeng. Biotechnol. 2:21. doi: 10.3389/fbioe.2014.00021

This article was submitted to Synthetic Biology, a section of the journal Frontiers in Bioengineering and Biotechnology.

Copyright (C) 2014 Davies, Work, Beliaev and Posewitz. This is an open-access article distributed under the terms of the Creative Commons Attribution License (CC BY). The use, distribution or reproduction in other forums is permitted, provided the original author(s) or licensor are credited and that the original publication in this journal is cited, in accordance with accepted academic practice. No use, distribution or reproduction is permitted which does not comply with these terms. 